\title{
Dual function of Arabidopsis glucan synthase-like genes GSL8 and GSL10 in male gametophyte development and plant growth
}

\author{
Armin Töller ${ }^{1, \dagger}$, Lynette Brownfield ${ }^{2, \dagger}$, Christina Neu ${ }^{1}$, David Twell ${ }^{2}$ and Paul Schulze-Lefert ${ }^{1, *}$ \\ ${ }^{1}$ Department of Plant-Microbe Interactions, Max-Planck-Institut für Züchtungsforschung, Carl-von-Linné-Weg 10, \\ D-50829 Köln, Germany, and \\ ${ }^{2}$ Department of Biology, University of Leicester, University Road, Leicester, LE1 7RH, UK
}

Received 6 December 2007; revised 31 January 2008; accepted 8 February 2008; published online 14 April 2008.

*For correspondence (fax +49 221 5062313; e-mail schlef@mpiz-koeln.mpg.de).

${ }^{\dagger}$ These authors contributed equally to this work.

\begin{abstract}
Summary
Members of the glucan synthase-like (GSL) family are believed to be involved in synthesis of the cell-wall component callose in specialized locations throughout the plant. We identified two members of the Arabidopsis GSL gene family, GSL8 and GSL10, that are independently required for male gametophyte development and plant growth. Analysis of $g s / 8$ and $g s / 10$ mutant pollen during development revealed specific malfunctions associated with asymmetric microspore division. GSL8 and GSL10 are not essential for normal microspore growth and polarity, but play a role in entry of microspores into mitosis. Impaired function of GSL10 also leads to perturbation of microspore division symmetry, irregular callose deposition and failure of generative-cell engulfment by the cytoplasm of the vegetative cell. Silencing of GSL8 or GSL10 in transgenic lines expressing gene-specific dsRNAi constructs resulted in a dwarfed growth habit, thereby revealing additional and independent wild-type gene functions for normal plant growth.
\end{abstract}

Keywords: callose synthase, pollen development, pollen mitosis, plant growth, cell-wall integrity, gametophytic lethality.

Introduction

Callose is a linear 1,3- $\beta$-glucan polymer with some 1,6 branches that is widespread in the cell walls of higher plants (Stone and Clarke, 1992). Callose deposits are formed during normal plant growth and development in sporophytic tissues including root hairs and the sieve plates of dormant phloem, at plasmodesmata and as a transitory component at the cell plate in dividing cells as well as in male and female gametophytes (Samuels et al., 1995; Stone and Clarke, 1992). Callose is also rapidly deposited in response to wounding or pathogen challenge (Stone and Clarke, 1992).

Callose is an important structural component in male gametophyte development, being deposited at several stages (McCormick, 1993; Stone and Clarke, 1992). Within the anther locule, the pollen mother cells are surrounded by callose before entry into meiosis. Following meiosis, callose is also deposited between the individual microspores during cytokinesis. This callose is important for exine patterning and is transient, being degraded to enable individual microspore release. The microspores then undergo an asymmetric division forming a large vegetative cell and a smaller generative cell, with callose forming a prominent but transient cell wall that separates the two cells (Park and Twell, 2001). The generative cell migrates into the vegetative cytoplasm where it undergoes a further mitotic division to produce two sperm cells. After pollen germination, the sperm cells are delivered to the ovule by the pollen tube, for which callose is a major component of the cell wall and the plugs that form along the length of the tube (Ferguson et al., 1998; Li et al., 1997; Nishikawa et al., 2005).

The 1,3- $\beta$-glucan polymer, which is highly impermeable, is also found in yeast and filamentous fungi, where it represents a major component of the cell wall. Formation of 1,3- $\beta$ glucan in Saccharomyces cerevisiae is dependent on the partially functionally redundant and sequence-related $F K S 1 /$ 
FKS2 genes that encode integral plasma membrane proteins that appear to serve as a substrate-binding catalytic subunit in heteromeric callose synthase complexes (Douglas et al., 1994; Mazur and Baginsky, 1996; Mazur et al., 1995; Schimoler-O'Rourke et al., 2003). Homologues of fungal FKS genes are found in higher plants, and comprise a family of 12 glucan synthase-like (GSL) genes in Arabidopsis (Hong et al., 2001; Saxena and Brown, 2000). Molecular and biochemical evidence supports a role for GSL proteins in callose synthesis (Brownfield et al., 2007; Cui et al., 2001; Hong et al., 2001; Li et al., 2003; Ostergaard et al., 2002), and recessive mutations in the few tested Arabidopsis GSL genes abolish either specific developmentally regulated or stress-induced callose deposits (Dong et al., 2005; Enns et al., 2005; Jacobs et al., 2003; Nishikawa et al., 2005; Nishimura et al., 2003), indicating potential functional diversification.

GSL5/PMR4 has been shown to direct the synthesis of wound- and pathogen-inducible callose in sporophytic tissue (Dong et al., 2005; Jacobs et al., 2003). In pollen development, GSL5 acts together with GSL1, and is required for formation of the callose wall that separates the microspores of the tetrad (Enns et al., 2005). The absence of GSL5 and GSL 1 renders pollen infertile, demonstrating that a gene family member can have unique as well as overlapping functions with another GSL family member in different biological processes. GSL2 acts during pollen development and is essential for formation of the callose wall surrounding pollen mother cells (Dong et al., 2005) and also contributes to the callose deposited in the wall and plugs of the growing pollen tube (Nishikawa et al., 2005).

To test the hypothesis that proliferation of GSL genes across the Arabidopsis genome reflects functional diversification of family members, we isolated T-DNA insertion mutants of GSL genes whose biological functions are unknown. Our failure to recover homozygous mutant plants for two independent T-DNA insertion alleles for GSL8 (gs/8-1 and $g s / 8-2$ ) and GSL10 (gs/10-1 and $g s / 10-2$ ) and the observed pollen developmental defects in all mutants demonstrate essential male gametophytic functions for the respective wild-type genes. Moreover, transgenic lines expressing 35S promoter-driven gene-specific dsRNAi constructs for GSL8 or GSL 10 exhibited a dwarfed growth habit, thereby revealing dual and independent functions of both genes in gametophyte and sporophyte development. Our findings suggest that plant GSL family members might exert indirect regulatory functions through interactions with other proteins rather than through their catalytic activity alone.

\section{Results}

\section{GSL8 and GSL10 have a gametophytic function}

For each of the highly sequence-related AtGSL8 (At2g36850) and AtGSL10 (At3g07160) glucan synthase-like family members, we isolated two independent T-DNA insertion lines (Figure 1). T-DNA lines SALK 111094 and GABI 851C04 contain an insertion in the GSL8 coding region close to its $5^{\prime}$ end and were named gs/8-1 and $g s / 8-2$, respectively. T-DNA lines GABI 038F11 and GABI 054E08 contain an insertion in the GSL10 coding region and were named gs/10-1 and gs/102 , respectively. DNA isolated from selfed progeny of the hemizygous T-DNA lines was examined by PCR analysis using T-DNA left border and gene-specific PCR primers to identify homozygous insertion lines. Among the progeny of each of the four tested insertion lines, we found only wildtype or hemizygous T-DNA lines, with a segregation ratio of about 1:1 (Table 1). There were no recognizable morphological differences between wild-type and hemizygous $g s / 8$

Table 1 T-DT-DNA segregation in progeny of selfed GSL8/gs/8 and GSL10/gs/10 mutant lines

\begin{tabular}{lllll}
\hline & $\begin{array}{l}\text { T-DT-DNA } \\
\text { Line }\end{array}$ & $\begin{array}{l}\text { No. } \\
\text { plants } \\
\text { tested }\end{array}$ & $\begin{array}{l}\text { Percentage } \\
\text { of plants } \\
\text { heterozygous }\end{array}$ & $\begin{array}{l}\text { Percentage } \\
\text { of plants } \\
\text { wild-type }\end{array}$ \\
\hline GSL8/gs/8-1-1 & SALK_111094 & 72 & 54.2 & 45.8 \\
GSL8/gs/8-2 & GK_851C04 & 76 & 63.2 & 36.8 \\
GSL10/gs/10-1-1 & GK_038F11 & 86 & 48.8 & 51.2 \\
GSL10/gs/10-2-2 & GK_054E08 & 78 & 53.8 & 46.2 \\
\hline
\end{tabular}

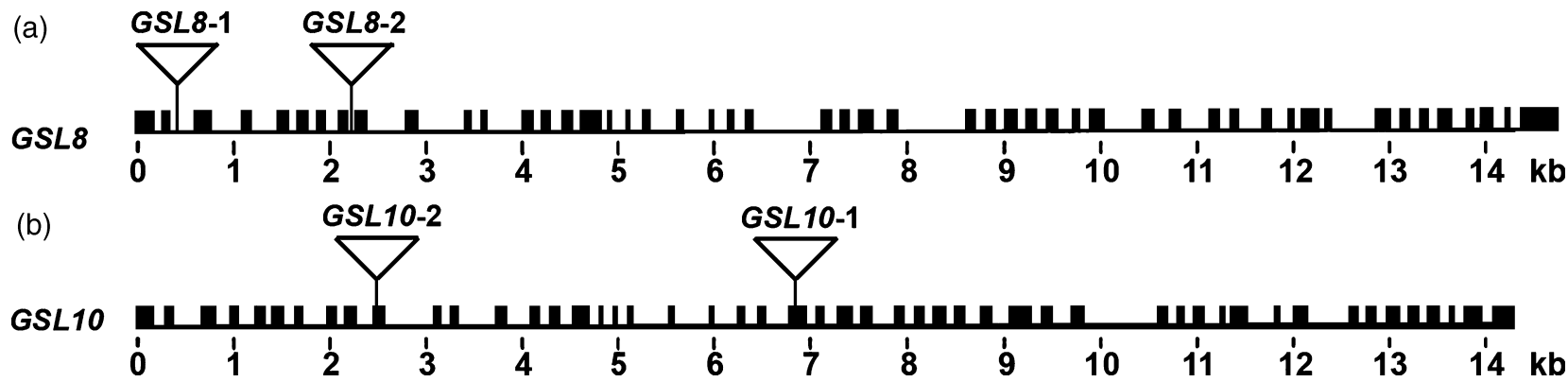

Figure 1. GSL8 and GSL10 gene structures.

Gene structures of (a) GSL8 and (b) GSL10. Black boxes represent exons and lines indicate introns. Triangles show the T-DNA insertion sites for the indicated gs/8 and $g s / 10$ mutant alleles. 
or gs/10 plants. The absence of homozygous T-DNA-containing progeny and the observed distorted segregation ratio of wild-type and hemizygous T-DNA plants provided initial evidence that homozygous $g s / 8$ and gs/10 mutants cannot be recovered due to essential gametophytic functions of the respective wild-type genes.

\section{GSL8 and GSL10 T-DNA insertions lead to pollen sterility}

As callose deposition is known to occur at multiple stages during male gametophyte development, we investigated pollen development in each hemizygous gs/8 and gs/10 TDNA line. Light microscopic analysis of mature pollen from GSL8/gs/8 plants revealed over $40 \%$ collapsed pollen, while pollen from GSL 10/gs/10 plants contained nearly $40 \%$ aberrant pollen, being either collapsed or mis-shapen (Table 2). In contrast, only $1-2 \%$ aberrant pollen was found in GSL8 or GSL10 wild-type siblings, and the increased frequency of aberrant pollen co-segregated with the respective T-DNA insertions. Closer examination of the collapsed pollen phenotype by scanning electron microscopy revealed a shrunken appearance of pollen grains, although the characteristic reticulate exine architecture of wild-type pollen was clearly visible in the collapsed grains (Figure 2). Thus both GSL8 and GSL10 are required for normal pollen development.

We investigated the expression of GSL8 and GSL10 during pollen development using RT-PCR with RNA isolated from spores at various developmental stages (Figure 3). Both GSL8 and GSL10 are expressed throughout pollen development, with GSL8 being most strongly expressed in bicellular and tricellular pollen and GSL10 showing peak expression in bicellular pollen. As GSL8 and GSL 10 are both expressed throughout pollen development, and mature pollen from GSL8/gs/8 and GSL10/gs/10 is aberrant, we conducted a detailed analysis of mutant pollen development to determine the role of GSL8 and GSL10 in male gametogenesis.

\section{GSL8 and GSL10 are not required for microspore development}

As extensive callose deposition is known to occur at the tetrad stage (McCormick, 1993), we visualized callose in the tetrads of the four hemizygous T-DNA insertion lines as well

Table 2 Light microscopic analyses of mature pollen from GSL8/ gs/8 and GSL10/gs/10 lines

\begin{tabular}{|c|c|c|c|c|c|}
\hline Line & $\begin{array}{l}\text { Total } \\
\text { pollen }\end{array}$ & $\begin{array}{l}\text { Collapsed } \\
\text { pollen }\end{array}$ & $\begin{array}{l}\text { Percentage } \\
\text { collapsed }\end{array}$ & $\begin{array}{l}\text { Aberrant } \\
\text { pollen }\end{array}$ & $\begin{array}{l}\text { Percentage } \\
\text { aberrant }\end{array}$ \\
\hline GSL8/gs/8-1 & 429 & 201 & 46.9 & 0 & 0 \\
\hline GSL8/gs/8-2 & 428 & 188 & 43.9 & 0 & 0 \\
\hline GSL 10/gs/10-1 & 562 & 50 & 8.4 & 171 & 30.4 \\
\hline GSL 10/gs/10-2 & 410 & 98 & 23.9 & 63 & 15.4 \\
\hline
\end{tabular}
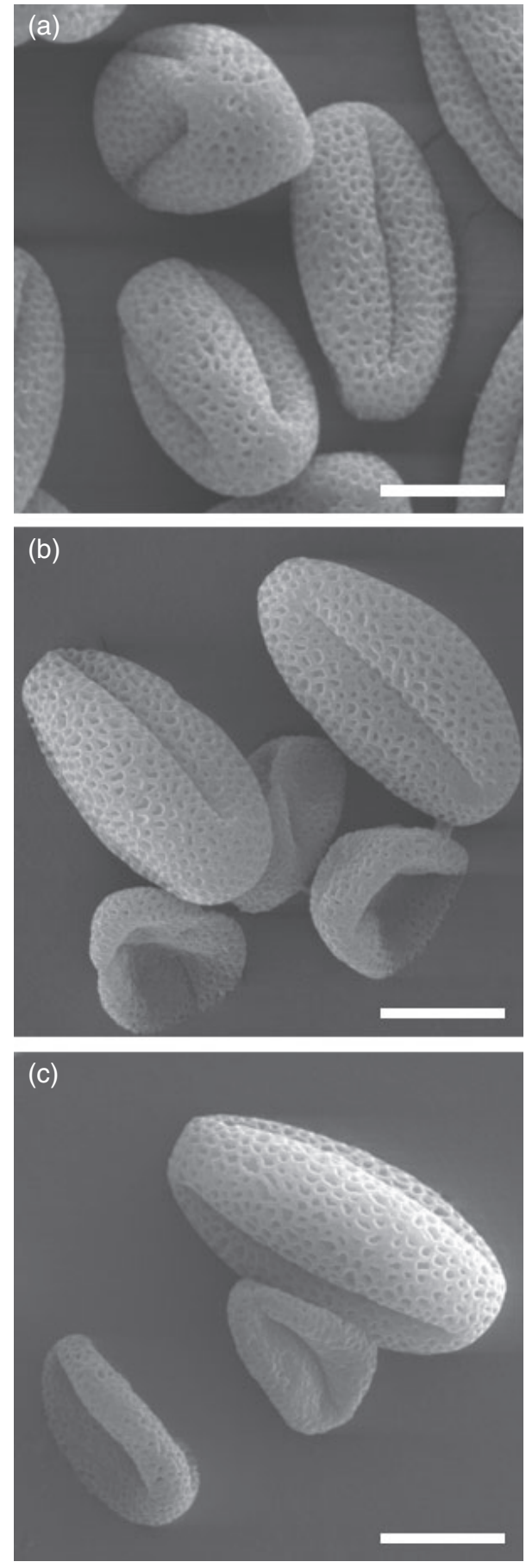

Figure 2. Aberrant $g s / 8$ and $g s / 10$ pollen phenotypes.

Scanning electron micrographs of pollen isolated from (a) wild-type, (b) GSL8/gs/8-1 and (c) GSL10/gs/10-2 mutant plants. Approximately half of the pollen grains of all tested heterozygous mutant lines exhibit an aberrant pollen phenotype. Bar $=10 \mu \mathrm{m}$.

as wild-type tetrads by aniline blue staining. We failed to detect either differences in callose deposition patterns or in the shape of haploid microspores between tetrads from 


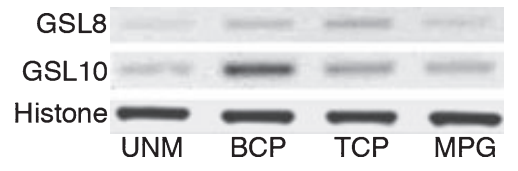

Figure 3. Expression of GSL8 and GSL10 during pollen development. RT-PCR analysis of GSL8 and GSL10 transcripts in uninucleate microspores (UNM), bicellular pollen (BCP), tricellular pollen (TCP) and mature pollen grains (MPG). A histone gene was used as a control.

wild-type and hemizygous GSL8/gs/8 and GSL10/gs/10 plants (Figure $4 \mathrm{a}-\mathrm{c}$ ). This might indicate that GSL8 and GSL10 activities are redundant during tetrad formation and/ or that both genes act gametophytically at a later stage of pollen development.

Older microspores were isolated and nuclear DNA was stained with DAPI. In buds containing free microspores, all spores of both wild-type and hemizygous T-DNA mutant lines exhibited a single, brightly stained centrally located nucleus (data not shown). During microspore development, the nucleus migrates to the microspore wall before the asymmetric cell division, producing a highly polarized cell (Figure $4 d$, arrow showing nucleus). In late-microsporestage anthers from a wild-type plant, approximately $90 \%$ of microspores were polarized (Figure 4e). Similarly, in both GSL8/gs/8 and GSL10/gs/10 plants, over $80 \%$ of the microspores were polarized (Figure 4e), demonstrating that GSL8- or GSL10-deficient microspores can still establish polarity.
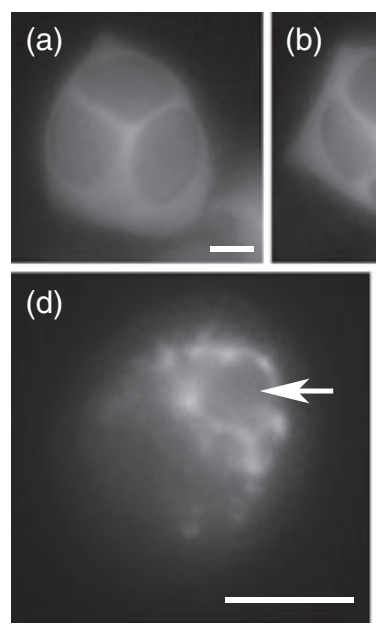

(e)

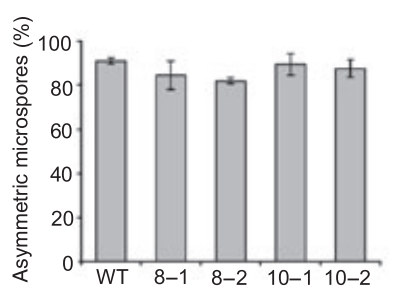

Figure 4. Microspore development is not interrupted in GSL8/gs/8-1 or GSL 10/gs/10-2 plants.

(a-c) Isolated microspore tetrads of (a) wild-type, (b) GSL8/gs/8-1 and (c) GSL10/gs/10-2 genotypes were stained with aniline blue. Callose deposition around and between microspores is indistinguishable between wild-type and mutant lines. Bars $=5 \mu \mathrm{m}$.

(d) WT microspore with a polarized nucleus indicated by an arrow. Bar $=10 \mu \mathrm{m}$.

(e) Microspores from GSL8/gs/8-1 (8-1), GSL8/gs/8-2 (8-2), GSL10/gs/10-1 (101) and GSL 10/gs/10-2 (10-2) become polarized to a similar level as WT.

\section{GSL8 and GSL10 exert essential functions associated with microspore division}

Pollen was also examined after completion of the asymmetric microspore division at the mid-bicellular stage when the generative cell had migrated into the vegetative cell cytoplasm (Figure $5 \mathrm{c}$ ). Only about half of the pollen from GSL8/gs/8-1 and GSL8/gs/8-2 plants was bicellular, in comparison to almost $100 \%$ of pollen from a wild-type plant at this stage (Figure 5a). Most of the remaining pollen had a single DAPI-staining nucleus, and organelles that stained with DAPI but were often slightly smaller and plasmolysed in comparison with bicellular pollen (Figure 5c,d). Moreover nuclei were sometimes enlarged or displayed disordered chromatin structure. This aberrant pollen phenotypic class was termed 'mononuclear', and indicates that GSL8 is required for microspore entry into mitosis. There was also a small amount of pollen with two similar DAPI-stained structures that resemble the vegetative nucleus with dispersed chromatin (Figure 5c,e), indicating that these microspores had undergone mitosis but cytokinesis had been disrupted.

In contrast, over $80 \%$ of pollen from the GSL $10 / \mathrm{gs} / 10-1$ mutant resembled that of wild-type pollen at the midbicellular stage (Figure 5a). Most of the remaining pollen was also bicellular, but, rather than migrating into the vegetative cell cytoplasm, the generative cell was positioned against the pollen wall, and was termed 'GC on wall' (Figure 5f). Approximately half the pollen from GSL10/ gs/10-2 plants was bicellular (Figure 5a). Of the remaining pollen, over half resembled $g s / 8$ pollen and was mononuclear, much contained two similar nuclei, and the generative cell was located against the wall in the remainder (Figure 5a). The mononuclear phenotype of gs/10-2 pollen indicates that, like GSL8, GSL10 is also involved in entry into mitosis and the similar nuclei class suggests that GSL10 is important for asymmetric division.

At later pollen stages, all pollen was tricellular in wild-type plants (Figure 5b), consisting of the vegetative cell enclosing two sperm cells (Figure $5 \mathrm{~g}$ ). Half the pollen from both GSL8/ gs/8-1 and GSL8/gs/8-2 plants was tricellular (the GSL8 portion of the pollen), while most of the remaining pollen had aborted (Figure $5 \mathrm{~b}$ ). In aborted pollen, the pollen grains had collapsed and there was no or only residual DAPI staining (Figure 5h). There were also small amounts of pollen that were still mononuclear or bicellular. This decrease in the proportion of aberrantly stained mononuclear-like microspores and the associated increase in the number of unstained and collapsed microspores probably reflects progressive cell death in gs/8 mutant microspores.

Similarly, at the tricellular pollen stage in GSL10/gs/10-1 and GSL10/gs/10-2 plants, only $50 \%$ of the pollen was tricellular (Figure 5b). The remaining pollen from GSL10/ 


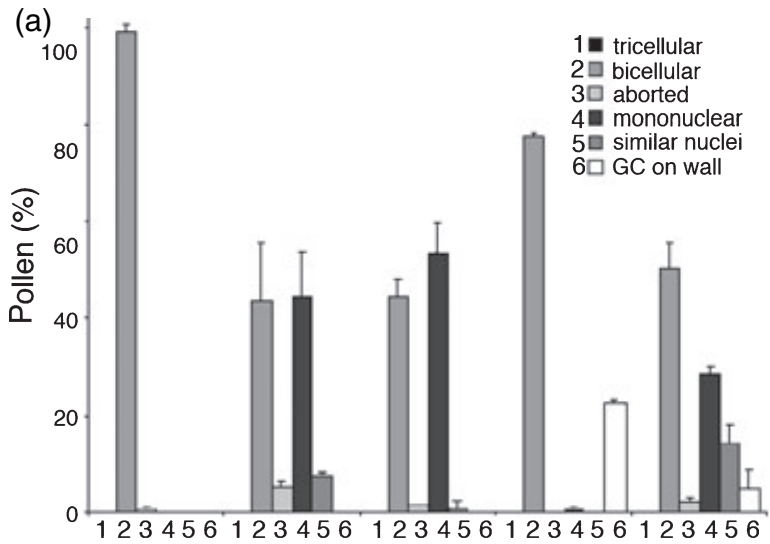

(b)
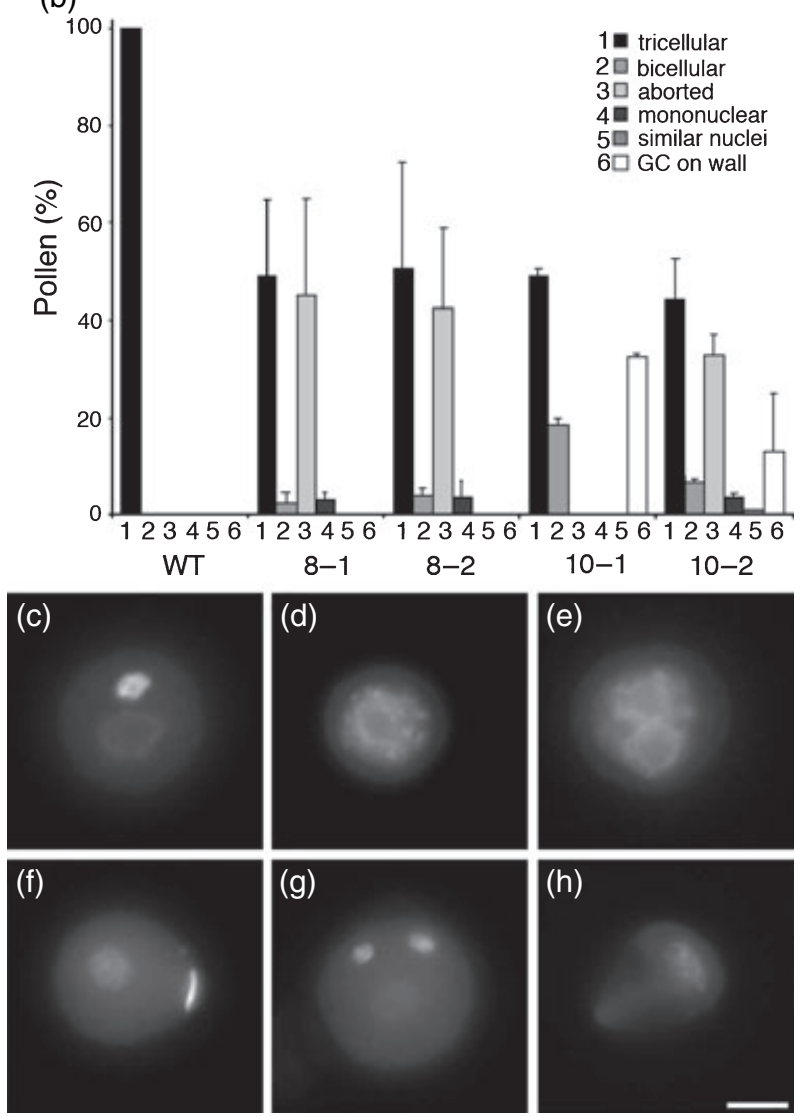

Figure 5. Phenotypes of bicellular and tricellular pollen from GSL8/gs/8 and GSL 10/gs/10 plants.

The phenotype of GSL8/gs/8-1 (8-1), GSL8/gs/8-2 (8-2), GSL10/gs/10-1 (10-1) and GSL 10/gs/10-2 (10-2) pollen at the mid-bicellular stage (a) and tricellular stage (b) was analysed in pollen in which the DNA had been stained using DAPI. Pollen phenotypes observed are (c) WT bicellular pollen, (d) mononuclear pollen, (e) pollen with two nuclei with a similar appearance (similar nuclei), (f) pollen with the generative cell stuck on the vegetative cell wall (GC on wall), (g) WT tricellular pollen and (h) aborted pollen. Bar $=10 \mu \mathrm{m}$.

gs/10-1 plants was bicellular, and in many cases the generative cell was tight against the wall (Figure 5b). The amount of pollen with the generative cell against the wall was higher in the tricellular pollen than at the bicellular stage, presum- ably because at the bicellular stage the generative cell was often close to the wall but not noticeably flattened. Much of the mutant pollen from GSL10/gs/10-2 plants had aborted by the tricellular stage (Figure $5 \mathrm{~b}$ ), and probably arose from pollen that was mononuclear or had two similar nuclei at the bicellular stage. The remaining pollen was bicellular, with the generative cell often tight against the wall, similar to that seen in GSL10/gs/10-1.

Collectively, these findings show that GSL8 is essential for microspore division, as most $g s / 8$ microspores fail to enter mitosis and subsequently abort. Some pollen from GSL10/ gs/10 also fails at mitosis entry (mainly in GSL10/gs/10-2), but a greater proportion of mutant pollen (mainly in GSL10/ gs/10-1) passes through microspore division but shows phenotypes that may relate to incorrect cytokinesis. The differences observed between gs/10-1 and gs/10-2 may relate to allelic differences. As only $50 \%$ of the pollen from hemizygous plants for each of the four T-DNA lines becomes tricellular, it appears that each line is fully penetrant and does not produce fertile pollen.

\section{Aberrant callose synthesis and degradation in gs/10 mutant pollen}

As GSL proteins are involved in callose synthesis, pollen from GSL8/gs/8 and GSL10/gs/10 plants was further analysed by co-staining for callose with aniline blue and for DNA with DAPI. In wild-type pollen, callose is transiently deposited after microspore mitosis between the newly formed generative and vegetative cell, and appears as a dome surrounding the generative cell (Figure $6 \mathrm{c}, \mathrm{d}$ ). In early bicellular pollen, this callosic dome can be detected in over $80 \%$ of pollen (Figure 6a). By the mid-bicellular stage (two buds older), virtually all aniline blue staining had disappeared, reflecting degradation of the callose wall (Figure 6b). This callose wall was not synthesized in aberrant GSL8/gs/8 pollen that failed to enter pollen mitosis I (data not shown). However, over $80 \%$ of early bicellular pollen from GSL10/ gs/10-1 plants contained callose in a dome over the generative cell (Figure 6a), indicating that these pollen grains can synthesize callose in a manner similar to wild-type. However, the callose appears to be more persistent, as approximately $20 \%$ of pollen at the mid-bicellular stage in GSL10/ gs/10-1 still contained an aniline blue-stained wall (Figure $6 \mathrm{~b}$ ). The persistence of the callose wall may therefore interfere with migration of the generative cell, possibly leading to the generative cell being tightly stuck against the wall later in development.

Aniline blue staining was also carried out on early bicellular pollen from GSL10/gs/10-2 plants. A callosic dome was observed in approximately $45 \%$ of early bicellular pollen (Figure 6a), suggesting that many gs/10-2 pollen grains fail to make a wild-type callosic wall. Ectopic callose was regularly observed (Figure 6e). This callose was generally 
(a)
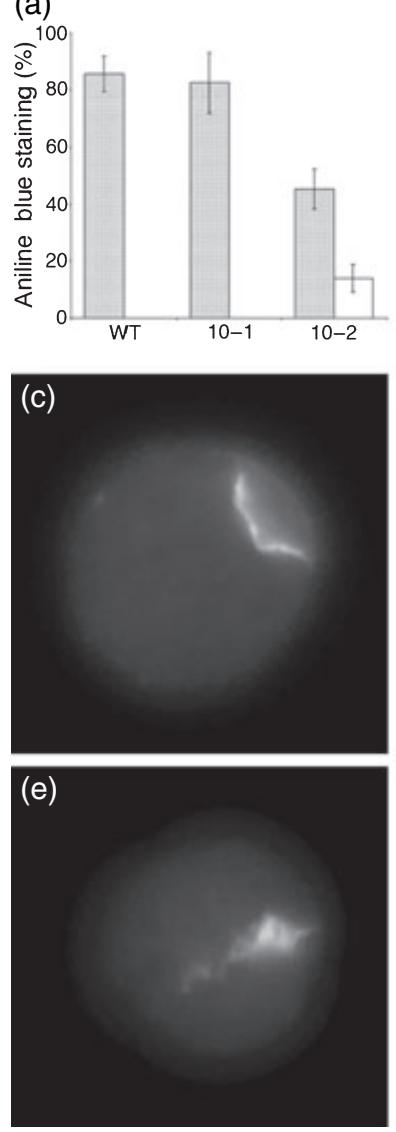

Aniline blue
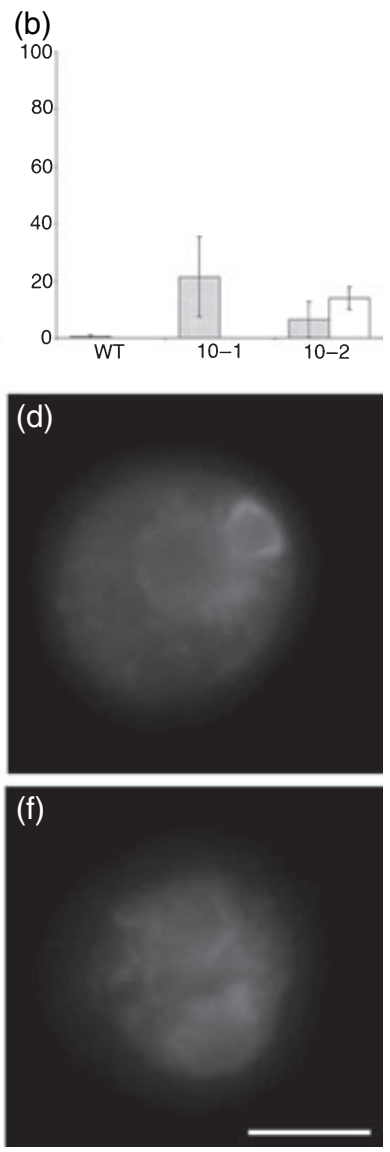

DAPI

Figure 6. Callose deposition in bicellular pollen from GSL10/gs/10 plants. Pollen from early bicellular (a) and mid-bicellular (b) stages was stained with aniline blue and DAPI. Shaded bars show the percentage of pollen with a WT callosic dome and empty bars indicate internal ectopic callose. In pollen from WT and GSL10/gs/10-1 plants, the majority of pollen has a dome-shaped callose wall (c) between the vegetative cell nucleus and the generative cell nucleus (d). In pollen from GSL10/gs/10-2 plants, internal ectopic callose is sometimes observed (e), often between two nuclei with a similar appearance (f). The same pollen grains are shown in (c) and (d) and in (e) and (f). Bar $=10 \mu \mathrm{m}$.

in a rough line, but not always complete, close to the centre of the pollen grain and often with regions where there appeared to be clumps of callose (Figure 6e). This

ectopic callose was often found (27/29) in pollen grains that also had two similar nuclei, and the callose was located between the two nuclei (Figure 6e,f). Ectopic callose was persistent in mid-bicellular pollen (Figure $6 \mathrm{~b}$ ) and was still present in pollen with two similar nuclei (25/27). A small amount of mid-bicellular pollen from GSL10/gs/10-2 plants also had a callosic dome (Figure 6b), similar to the persistence of aniline blue staining in GSL10/gs/10-1 pollen, suggesting some gs/10-2 pollen do make a callosic dome that persists; this could also result in the generative-cell marginalization observed in tricellular stage pollen from these plants.

\section{Transmission electron microscopy of pollen phenotype}

To further analyse the phenotype of the mutant $g s / 8$ and gs/10 pollen, buds containing early to mid-bicellular pollen and buds containing tricellular pollen from GSL8/gs/8 and GSL 10/gs/10 plants were examined by transmission electron microscopy (TEM). We observed two distinct classes of pollen from GSL8/gs/8 plants in an anther locule containing early to mid-bicellular pollen (Figure 7a). A portion of pollen had dense cytoplasm including a generative and vegetative cell (Figure 7b), similar to wild-type pollen (Figure 7e-h). The remaining pollen had less dense cytoplasm, disrupted cellular structure and a single, sometimes abnormally shaped, nucleus (Figure 7c,d). Such pollen grains are not seen in wild-type and are likely to be the gs/8 mutant pollen classified as mononuclear in Figure 5.

Unlike GSL8/gs/8, the pollen in a bicellular-stage anther from GSL 10/gs/10-1 had a density of cytoplasmic staining similar to that of the wild-type (Figure 7i,e). The gs/10-1 mutant pollen often appears to have a callose wall separating the generative and vegetative cells that, under aniline blue staining, appears similar to the wall in wild-type pollen but persists (Figure 6b). The ultrastructure of the wall in pollen from GSL10/gs/10-1 plants (Figure 7j-I) was compared with that of wild-type pollen (Figure $7 f-h$ ). In wild-type pollen, the callosic wall is generally smooth and forms a dome around the generative cell (Figure 7f). During the process of generative-cell detachment, the callosic wall becomes thinner in the central region with thickened stubs

Figure 7. TEM analysis of pollen phenotypes.

Pollen from anthers at the early to mid-bicellular stage from GSL8/gs/8 plants (a-d), wild-type plants (e-h) and GSL 10/gs/10-1 plants (i-l). Pollen from tricellular stage anthers from GSL10/gs/10-1 (m-p) and GSL10/gs/10-2 plants (q-t).

(a) Anther locule from GSL8-1/gs/8-1 plant at the early to mid-bicellular stage with light and dark staining of pollen.

(b) Wild-type bicellular pollen grain from $G S L 8 / g / s 8-1$ bud.

(c, d) Examples of reduced cytoplasmic density in (c) gs/8-1 and (d) gs/8-2 pollen.

(e) Anther locule from a wild-type plant showing consistent cytoplasmic staining.

( $f-h)$ Wild-type pollen grain with smooth domed-shaped wall (arrow) between the newly divided generative and vegetative cells ( $f$ ) and the generative cell migrating off the vegetative wall with rounded generative cells and callose wall stubs (arrow) $(\mathrm{g}, \mathrm{h})$.

(i) Anther locule from a GSL10/gs/10-1 plant with consistent cytoplasmic staining.

(j-l) Examples of flattened and disrupted wall (arrow) between the generative and vegetative cells (j, k) and ectopic callose (arrow) in the GSL10/gs/10-1 mutant (I). $(\mathrm{m}, \mathrm{q})$ Anther locules from GSL 10/gs/10-1 (m) and GSL10/gs/10-2 (q) showing wild-type and mutant pollen.

$(n, r)$ Wild-type pollen grains from tricellular-stage sperm cells, indicated by arrows; only one sperm cell is visible in ( $n$ ).

$(0, p, s, t)$ Examples of the phenotypes generative cell stuck on wall $(o, s)$, partial walls $(p)$ and two similar nuclei $(t)$. 
at junctions with the outer pollen grain wall, while the generative cell becomes more rounded (Figure 7g,h). In some pollen from GSL10/gs/10-1 plants, the wall separating the generative and vegetative cells was disturbed, being flattened rather than consistently dome-shaped, and the wall was not always smooth and showed some
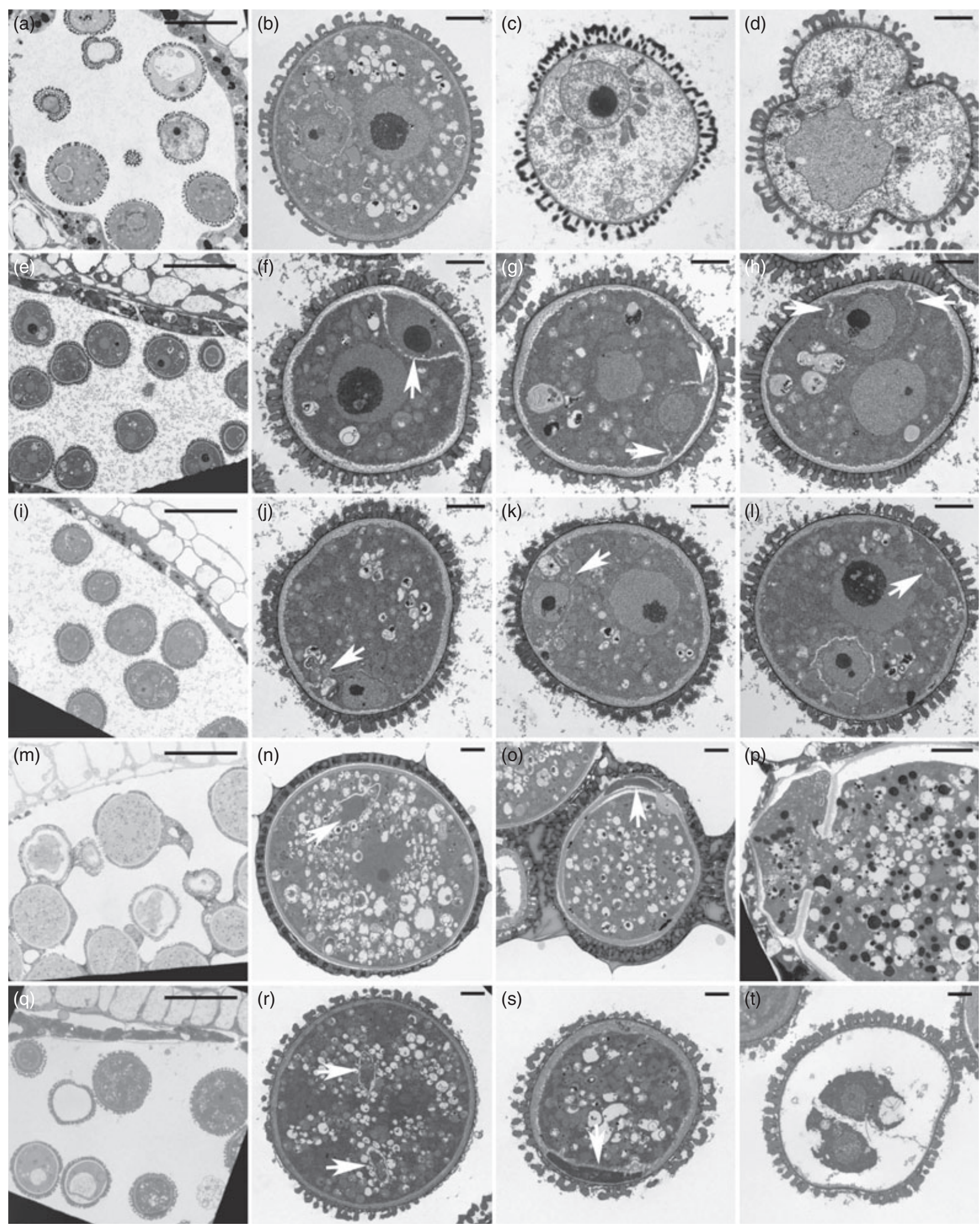

(C) 2008 The Authors

Journal compilation (c) 2008 Blackwell Publishing Ltd, The Plant Journal, (2008), 54, 911-923 
invaginations (arrows in Figure 7j,k). Ectopic internal walls were also observed that were not located between the generative and vegetative cells (Figure 7l). Such ectopic walls may also have contributed to the number of persistent walls with a wild-type appearance that were seen with aniline blue staining (Figure $6 \mathrm{~b}$ ).

Pollen was also observed from buds at the tricellular stage from GSL10/gs/10-1 (Figure 7m-p) and GSL10/gs/10-2 plants (Figure 7q-t). Pollen was not observed for the gs/8 mutants because previous analysis had shown that the mutant pollen was aborted by this stage. Anther locules from GSL10/gs/10-1 and GSL10/gs/10-2 plants contained a mixture of pollen with dense cytoplasm filling the entire grain, and slightly smaller pollen grains where the cytoplasm was reduced or absent (Figure $7 \mathrm{~m}, \mathrm{q}$ ). The wild-type pollen at this stage has dense cytoplasm with a vegetative nucleus and two sperm cells (Figure $7 n, r$ ). In both mutants, pollen grains with a marginalized, electron-dense region separated from the main cytoplasm by wall-like material were present (Figure 7o,s), and presumably represent the class of pollen with the generative cell stuck on the wall that was seen with DAPI staining (Figure $5 \mathrm{~b}$ ). In some cases, the wall separating this region was incomplete (Figure $7 p$ ). Pollen with two similar nuclei was also seen in the GSL10/ gs/10-2 mutant, although by this stage much of the cytoplasm is degraded (Figure 7t), consistent with abortion of this class of pollen (Figure $5 a, b$ ).

\section{GSL8 and GSL10 act independently in the sporophyte}

The expression of GSL8 and GSL10 in diverse sporophytic tissues and organs (Zimmermann et al., 2004; Figure S1) prompted us to examine potential roles of GSL8 and GSL10 in sporophyte development. As homozygous gs/8 or gs/10 mutant plants cannot be recovered, we chose a gene silencing approach to downregulate GSL8 or GSL10 using 35S promoter-driven gene-specific dsRNAi constructs. As GSL5 was previously shown to function both in the male gametophyte and in sporophytic tissue (Jacobs et al., 2003; Nishimura et al., 2003), the resulting dsRNAi constructs were transformed into the $g s / 5$ (pmr4-1) mutant background to examine potential gene interactions between GSL5 and GSL8 or GSL10. We recovered independent dsRNAi lines that showed a strong reduction of GSL8 or GSL10 leaf transcript levels (Figure 8a). Although GSL8 and GSL10 are more closely related to each other than to any other GSL family member $163.7 \%$ DNA sequence identity, $63.4 \%$ protein sequence identity), the silencing effect appeared to be gene-specific as indicated by semiquantitative RT-PCR analysis of the respective dsRNAi lines (Figure 8a). dsRNAi lines containing a single locus integration event in the $g s / 5$ background were back-crossed with Col-0 wild-type plants to produce $F_{2}$ populations. Plants that were Basta-resistant (indicating the presence of the dsRNAi transgene) were screened using a cleaved amplified polymorphism marker to determine whether these were GSL5/GSL5 (WT), gs/5/gs/5 or GSL5/gs/5 (Table 3). The effectiveness of GSL8 or GSL10 dsRNAimediated gene silencing, measured by semi-quantitative RT-PCR analysis of the respective endogene-derived mRNA levels, was found to be indistinguishable in $G S L 5$ or $g s / 5$ backgrounds (Figure $8 \mathrm{a}, \mathrm{b}$ ).

In GSL5 wild-type and gs/5 mutant backgrounds, we noticed a marked dwarf phenotype in both GSL8 and GSL10 dsRNAi lines. The dwarf phenotypes of GSL8 or GSL10 dsRNAi-containing siblings were enhanced in the gs/5 background (compare Figure $8 \mathrm{~g}-\mathrm{j}$ with Figure $8 \mathrm{k}-\mathrm{p}$ ). Dwarfism in the GSL8 dsRNAi lines was consistently less severe compared with the GSL10 dsRNAi lines in wild-type and mutant gsl5 backgrounds (Figure $8 \mathrm{c}, \mathrm{d}, \mathrm{g}-\mathrm{j}$ ). These data demonstrate that GSL8 and GSL10 are independently required for normal growth of wild-type plants. Consistent with previous findings (Enns et al., 2005), homozygous gs/5 mutants were slightly smaller compared with wild-type plants.

We could discriminate dwarf subtypes for both GSL8 and GSL 10 dsRNAi lines among $T_{2}$ progeny in the $g s / 5$ background, designated 'moderate' or 'extreme' (compare Fig ure $8 \mathrm{l}, \mathrm{m}, \mathrm{o}, \mathrm{p})$. To test whether the severity of dwarfism was dependent on transgene dosage, we examined the segregation of dwarf phenotypes. As viable seeds of extreme dwarfs were difficult to recover in sufficient quantities, we restricted our analysis to $T_{3}$ progeny derived from moderately dwarfed $\mathrm{T}_{2}$ plants with single locus insertions. Among the Basta-resistant $T_{3}$ progeny, we found moderate and extreme dwarfs in a ratio of approximately $2: 1$, which might indicate a dose-dependent effect of the dsRNAi transgene on the severity of dwarfed plant growth.

\section{Discussion}

To investigate the functional diversification and roles of GSL family members in Arabidopsis, two independent T-DNA insertion lines each for GSL8 (gs/8-1 and gs/8-2) and GSL 10 (gs/10-1 and gs/10-2) were characterized. We have shown that both GSL8 and GSL10 are required gametophytically for pollen development, and both GSL proteins also have a role in the sporophyte.

Previously reported mutations in genes of the glucan synthase-like family that act in male gametogenesis affect tetrad callose deposition and/or exine architecture. GSL2 plays an essential role in callose synthesis at the primary cell wall of meiocytes, tetrads and microspores, and is required for correct exine patterning (Dong et al., 2005). GSL1 and GSL5 play partially redundant roles in pollen development, and are responsible for formation of the callose wall that separates the microspores of the tetrad (Enns et al., 2005). Unlike these GSL family members, GSL8 and GSL10 are not 
Figure 8. Silencing of GSL8 and GSL10 in wildtype and $g s / 5$ mutant backgrounds. (a) Semi-quantitative RT-PCR of GSL8 and GSL10 transcripts in wild-type (WT), gs/5 mutant plants, and transgenic lines expressing gene-specific GSL8 (GSL8i) and GSL10 (GSL10i) dsRNAi constructs in the $g s / 5$ background.

(b) Semi-quantitative RT-PCR of GSL8 and GSL10 transcripts in wild-type (WT), gs/5 mutant plants, and transgenic lines expressing gene-specific GSL8 (GSL8i) and GSL10 (GSL10i) dsRNAi constructs in the $G S L 5$ background.

(c, e, g, i, k, n) Top views of 28-day-old plants of the indicated genotypes. Bars $=1 \mathrm{~cm}$.

(d, f, h, j, l, o) Side views of 49-day-old plants of the indicated genotypes. Bars $=3 \mathrm{~cm}$.

$(\mathrm{m}, \mathrm{p})$ Top views of 49-day-old extreme dwarfs of the indicated genotypes. Bar $=1 \mathrm{~cm}$.
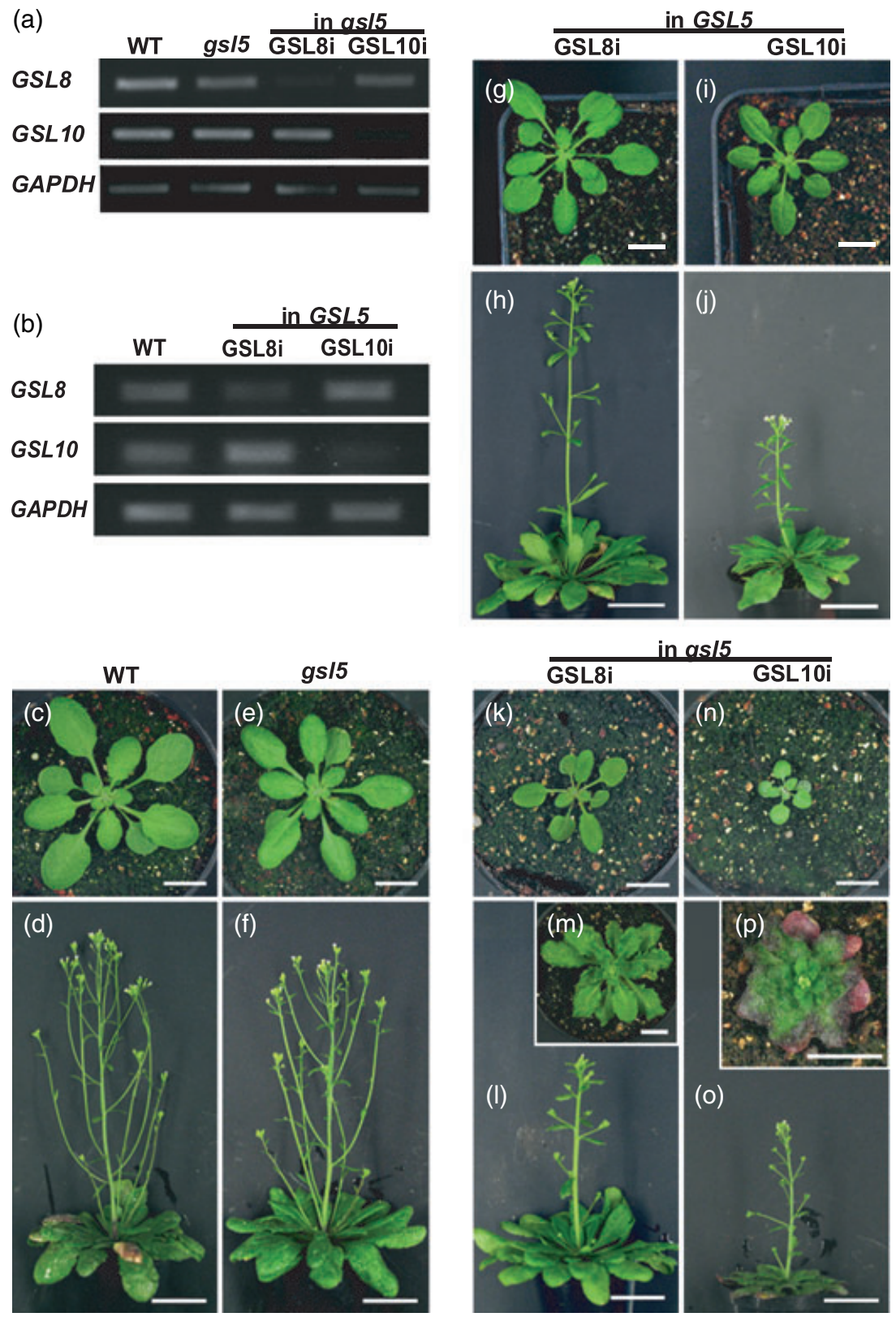

in $9 s / 5$

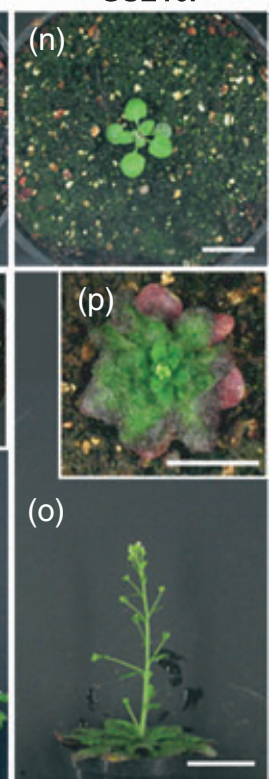

Table 3 Segregation of dsRNAi transgenes in $\mathrm{F}_{2}$ progeny of crosses between GSL8 or GSL10 dsRNAi transgenic lines (in gs/5 background) and Col-0 wild-type

\begin{tabular}{llllll}
\hline & $\begin{array}{l}\text { No. } \\
\text { dsRNAi }\end{array}$ & $\begin{array}{l}\text { No. } \\
\text { plants } \\
\text { line }\end{array}$ & $\begin{array}{l}\text { Norta } \\
\text { werminated } \\
\text { survivors }\end{array}$ & $\begin{array}{l}\text { wo. } \\
\text { type }\end{array}$ & $\begin{array}{l}\text { gs/5 } \\
\text { seterozygous }\end{array}$ \\
\hline GSL8i \#1 & 100 & 76 & 21 & 18 & 37 \\
GSL10i \#1 & 97 & 72 & 19 & 12 & 41 \\
GSL10i \#2 & 98 & 69 & 21 & 18 & 37 \\
\hline
\end{tabular}

required for microspore development, as mutant pollen has a reticulate exine, callose deposition in tetrads appears normal, and microspores become polarized in preparation for asymmetric division. These previously reported GSL family members also act later in pollen development. GSL2 is required for callose deposition in growing pollen tubes (Nishikawa et al., 2005), while gs/1/gs/5 mutant pollen are reported to have a bicellular pollen phenotype (Enns et al., 2005). GSL8 and GSL10 also act during pollen development and independently have a role in the entry of polarized microspores into mitosis. Perturbation of generative-cell engulfment, which involves spatially controlled synthesis and turnover of the separating callose wall, was mainly observed in gs/10-2 pollen.

Relatively few gametophytic mutants have been described in Arabidopsis that affect defined steps of micro- 
gametogenesis (Twell et al., 2006). Mutants and genes affecting microspore division symmetry and cytokinesis (Oh et al., 2006; Park et al., 1998; Twell et al., 2002) or that specifically block generative cell division have been isolated (Durbarry et al., 2005), but none that specifically block microspore division. $g s / 8$ and $g s / 10$ therefore represent a new class of gametophytic mutants that act during microspore development to prevent mitotic entry. Interestingly, the limpet pollen mutant, in common with $g s / 10$, also fails to complete generative-cell migration after microspore division (Howden et al., 1998), although the locus responsible remains unknown.

In both hemizygous $g s / 8$ and $g s / 10$ (mainly gs/10-2) lines, some mutant pollen displays a single DAPI-stained nuclei followed by progressive cell death (Figure 5a,b), indicating a role for GSL8 and GSL10 in entry into mitosis. Such a role for a GSL protein is intriguing and has not been shown before. Callose deposition before entry into mitosis, in the microspore or other cell types, has not been described. Thus the functions of GSL8 and GSL10 at this stage may be independent of their functions in callose synthesis. GSL8 and GSL10 may exert indirect regulatory functions through interactions with other proteins rather than through cataIytic activity at this stage. Whether this regulatory function involves surveillance of cell-wall integrity systems similar to those described in yeast in which FKS1/2 represent effector proteins remains to be tested (reviewed by Levin, 2005).

When gs/8 (rarely) or gs/10 microspores do divide, there are commonly defects in cytokinesis. Callose deposition was previously shown to occur during cytokinesis in all higher plants (Scherp et al., 2001), where it consolidates the tubular network into a new cell plate (Samuels et al., 1995). In gs/10 mutant pollen, callose is still deposited but either in the wrong location in an irregular structure (mainly gs 10-2) or as a dome surrounding the generative cell but with slight structural abnormalities (observed by TEM, Figure 7) that persists (mainly in $g s / 10-1$ ), indicating that GSL10 is not essential for callose production during pollen mitosis but may be required for correct temporal and spatial control of its deposition. GSL6 has been reported to be important in the cell plate between dividing cells in sporophytic tissues (Hong et al., 2001). Whether GSL6 is also involved in the production of callose after microspore division, and contributes to the callose deposited in gs/10 mutant pollen, is unknown.

Although all four T-DNA lines show similar pollen phenotypes, the proportions of pollen that fail to enter mitosis or display cytokinetic phenotypes differ. The majority of gs/8 mutant pollen fails to enter mitosis, indicating a requirement for GSL8 in this process and consistent with both $g$ s/8 alleles being null as the insertions occur close to the $5^{\prime}$ end of the coding region (Figure 1). The gs/10-2 insertion, which results in some mononuclear pollen, also occurs close to the $5^{\prime}$ end of GSL10 so is also likely to be a null allele, suggesting that the requirement for GSL8 in microspore entry into mitosis is not absolute. The proportion of $g s / 10-1$ and $g s / 10-2$ pollen in the various phenotypic classes differs and may be due to allelic differences. Consistent with this, the T-DNA insertion in $g s / 10-1$ is located almost exactly in the middle of the $14 \mathrm{~kb}$ GSL10 gene, thus potentially giving rise to a truncated gene product with residual activity (Figure 1). As the gs/10-1 T-DNA insertion is located upstream of the region encoding the putative catalytic region (deduced amino acids 10641796) that is conserved among GSL family members, the residual GSL10-1 activity most likely lacks callose synthase activity. That there appears to be some function of GSL10 while lacking the putative catalytic domain is consistent with the hypothesis that these GSL proteins may have a function other than callose synthesis in entry into mitosis.

Both GSL8 and GSL10 are expressed throughout pollen development, and their products may have roles after microspore division, in tricellular pollen for example, that are not apparent in these T-DNA lines. Several alleles of GSL2 have been described: $g s / 2-1$ and $g s / 2-2$ both result in microspore collapse (Dong et al., 2005), but a weaker allele gs/2-3 revealed a role for GSL2 in callose deposition during pollen tube growth (Nishikawa et al., 2005). Analysis of further alleles for GSL8 and GSL10 may also reveal further roles for these proteins that may be masked by earlier phenotypic defects in strongly defective alleles.

In addition to their role in male gametophyte development, we have shown that GSL8 and GSL10 are independently required for normal plant growth (Figure 8). If the cellular function(s) of GSL8 and GSL10 in male gametophyte and sporophytic cells are conserved, then dwarfism in GSL8 and GSL10 dsRNAi plants could be the consequence of partially impaired entry into mitosis or cytokinesis defects. Alternatively, the dwarf phenotypes that are enhanced in the $g s / 5$ background may arise from de-regulation of the salicylic acid pathway. GSL5/ PMR4 has been shown to negatively regulate the salicylic acid pathway (Nishimura et al., 2003), and de-regulated salicylic acid levels can dramatically affect cell growth (Rate et al., 1999; Vanacker et al., 2001; Yang and Hua, 2004).

A meta-analysis of 1388 publicly available genome-wide gene expression profiling experiments reveal highly correlated gene expression for GSL8 and GSL10 (Pearsons's correlation coefficient $r=0.66$; http://www.atted.bio.titech. ac.jp). This and the similar mutant $g s / 8$ and gs/10 phenotypes in both the gametophyte and sporophyte indicate related or overlapping gene functions, and suggest potential concerted co-action of these two most closely related GSL family members. Callose synthases may exist as 
complexes containing a number of catalytic domains as callose usually exists as triple helices (Pelosi et al., 2003; Stone and Clarke, 1992), and GSL proteins are present in very-high-molecular-weight complexes on native gels (Kjell et al., 2004; Li et al., 2003). Thus, it is tempting to speculate that the strikingly similar phenotypes resulting from lossof-function mutations in GSL8 or GSL10 during microspore mitosis as well as during sporophyte development reflect the existence of a GSL8-GSL10 heteromeric callose synthase-like complex in which the absence of one family member disrupts complex activity. This would also explain why both gene functions are genetically independent. Such heteromeric complexes have been shown for cellulose synthase (CESA) complexes, with three different CESA proteins being present in a complex (Gardiner et al., 2003; Taylor et al., 2000).

\section{Experimental procedures}

\section{Plant material and growth conditions}

Plants were grown under a $8 \mathrm{~h}$ light period and at $23^{\circ} \mathrm{C}$ for 5 weeks in Voetsch phytochambers (http://www.voetsch.info/) and subsequently shifted to a greenhouse. Pictures were taken 4 and 7 weeks after germination. The Arabidopsis thaliana GSL T-DNA insertion lines were the GSL8 insertions SALK_111094, provided by SIGnAL (http://www.signal.salk.edu/) and GABI_851C04, provided by GABIKat (Alonso et al., 2003; Rosso et al., 2003), and the GSL10 insertions GABI_038F11 and GABI_054E08, both provided by GABI-Kat. PCR primers specific for T-DNA left borders were $5^{\prime}$-ATATTGACCATCATACTCATTGC-3' (GABI-Kat) or 5'-TGGTTCACGTAGTGGGCCATCG-3' (SALK). The GSL-specific oligonucleotides used for T-DNA insertion detection by PCR were $5^{\prime}$-GGCAAGAACTTGGACCCTA- $3^{\prime}$ (GSL08-SALK_111094), 5'-TGCAGAGACGATCAGATGCT-3' (GSL8GABI_851C04), 5'-TGAACCAATTCGAGCCTACC-3' (GSL10-GABI_ 038F11) and 5'-TGTAATGGAACCCATCAAGAAA-3' (GSL10-GABI_ 054E08). The powdery mildew resistance line pmr4-1 was obtained from S. Sommerville (Carnegie Institution of Washington, Stanford, CA). PMR4 is identical with GSL5 (Jacobs et al., 2003). The pmr4-1 mutation was identified by Nhel digestion of a genomic PCR amplification product using the gene-specific primers $5^{\prime}$-CAAGGACGGCATTCATAGGT-3' and 5'-CCGTCTCGCCTCTAGATTCA-3'.

\section{dsRNAi constructs}

Gene-specific fragments were inserted into the plasmid pJawohl8 (AF408413) to create p8-08i (GSL8 At2g36850) and p8-10i (GSL10 At3g07160) dsRNAi constructs. The binary pJawohl8 dsRNAi vector harbors two Gateway ${ }^{\circledR}$ conversion cassettes (Invitrogen Corporation; http://www.lifetech.com/content/sfs/manuals/11797016.pdf) in sense and antisense orientations separated by intron 1 of the Arabidopsis thaliana transcription factor gene WRKY33 (GeneBank accession AF408413). Primer combinations to produce the genespecific GSL dsRNAi fragments were as follows: $5^{\prime}$-XATGCTGCACTAAACGCACAG-3' and 5'-XGGCGATCGGAGAAAATATGA-3' for GSL8 (745 bp product) and 5'-XGTACCTTGCGTATGGCAATGA-3' and ' 5'-XTAGCCTTGCTAGGGAACGAAT- $3^{\prime}$ for GSL10 (659 bp product). $X$ denotes forward or reverse Gateway ${ }^{\circledR}$ recombination sites. Plants were transformed by the floral-dip method (Clough and Bent, 1998) using Agrobacterium tumefaciens strain
GV3101::pMK(90). Transgenic seedlings expressing dsRNAi constructs were selected by spraying with BASTA ${ }^{\circledR}$ (http://www. bayercropscience.de) twice within 1 week.

\section{$R T-P C R$ analysis}

RNA was isolated from Arabidopsis leaves using the RNeasy Plant Mini Kit (Qiagen, http://www.qiagen.com/) or from spores at various stages of development as described previously (Honys and Twell, 2004). First-strand cDNA was synthesized from $0.5 \mu \mathrm{g}$ of total RNA extract using the Superscript II cDNA synthesis kit (Invitrogen, http:// www.invitrogen.com/). PCR ( 25 cycles) was performed using $2 \mu \mathrm{l}$ of 1:1 diluted first-strand reaction with gene-specific primers to identify endogenous GSL8 (5'-GGACTTAACCAGATTGCCCTA-3' and 5'-ATACCTTTGCACCACCGTGA-3') and GSL10 (5'-GAGATGTTGGGCTCAATCAAA- $3^{\prime}$ and 5'-CATTGGCACAGCAGTGAAGA-3'). Additional gene-specific primers were used to amplify endogenous glyceraldehyde-3-phosphate dehydrogenase (GAPDH; 5'-AGCTCGTCGCTGTCAACG- $3^{\prime}$ and $5^{\prime}$-GACAGCCTTGGCAGCTCC- $3^{\prime}$ ) in a PCR reaction with 22 cycles as an internal control. For spore stages, PCR was performed with $1 \mu \mathrm{l}$ of a 1:10 dilution of the first-strand reaction solution using primers $5^{\prime}$-TTTTCTCTCGGGACAAGAACTC- 3 ' and 5'-CTTTACTCCCACTCCACCTTTG-3' for GSL8 and 5'-ACCTTGAGCGGGAGGATAAT-3' and '5'-GGGTGTGAGCACAACTGCTA-3' for GSL10, with a histone gene (At4g40040) as a control (primers 5'-AGCTCCCTTTCCAGAGGCTA-3' and 5'-TCCAAGTCTCCTACACCCAAA-3')

\section{Microscopy}

For analysis of spores during development, anthers were dissected and spores stained with DAPI (4,6-diamidin-2-phenylindol-dihydrochlorid) and $0.03 \%$ aniline blue as described previously (Park et al., 1998). For each line, spores from buds from two or three inflorescences were analysed, with 80-200 spores observed per bud. The results are presented as the mean percentage of the pollen for each bud.

\section{Scanning electron microscopy}

Pollen grains were sputter coated with gold and observed using a Zeiss DSM 940 scanning electron microscope (http://www. zeiss.com/). Transmission electron microscopy was performed as described previously (Park and Twell, 2001).

\section{Acknowledgements}

We thank Natalie Allcock of the University of Leicester Electron Microscopy Laboratory for transmission electron microscopy. A.T. was supported by a fellowship from the International Max Planck Graduate Research School (IMPRS). P.S.-L. and C.N. thank the Bundesminsterium für Bildung und Forschung (GABI Nonhost Resistance Consortium) for financial support. D.T. and L.B. thank the UK Biotechnology and Biological Sciences Research Council for financial support.

\section{Supplementary Material}

The following supplementary material is available for this article online:

Figure S1. Expression pattern of GSL8 and GSL10. 
This material is available as part of the online article from http:// www.blackwell-synergy.com.

Please note: Blackwell publishing are not responsible for the content or functionality of any supplementary materials supplied by the authors. Any queries (other than missing material) should be directed to the corresponding author for the article.

\section{References}

Alonso, J.M., Stepanova, A.N., Leisse, T.J. et al. (2003) Genomewide insertional mutagenesis of Arabidopsis thaliana. Science, 301, 653-657.

Brownfield, L., Ford, K., Doblin, M.S., Newbigin, E., Read, S. and Bacic, A. (2007) Proteomic and biochemical evidence links the callose synthase in Nicotiana alata pollen tubes to the product of the NaGSL1 gene. Plant J. 52, 147-156.

Clough, S.J. and Bent, A.F. (1998) Floral dip: a simplified method for Agrobacterium-mediated transformation of Arabidopsis thaliana. Plant J. 16, 735-743.

Cui, X., Shin, H., Song, C., Laosinchai, W., Amano, Y. and Brown, R.M. Jr (2001) A putative plant homolog of the yeast $\beta$-1,3-glucan synthase subunit FKS1 from cotton (Gossypium hirsutum L.) fibers. Planta, 213, 223-230.

Dong, X., Hong, Z., Sivaramakrishnan, M., Mahfouz, M. and Verma, D.P. (2005) Callose synthase (CalS5) is required for exine formation during microgametogenesis and for pollen viability in Arabidopsis. Plant J. 42, 315-328.

Douglas, C.M., Foor, F., Marrinan, J.A. et al. (1994) The Saccharomyces cerevisiae FKS1 (ETG1) gene encodes an integral membrane protein which is a subunit of 1,3- $\beta$-D-glucan synthase. Proc. Natl Acad. Sci. USA, 91, 12907-12911.

Durbarry, A., Vizir, I. and Twell, D. (2005) Male germ line development in Arabidopsis. duo pollen mutants reveal gametophytic regulators of generative cell cycle progression. Plant Physiol. 137, 297-307.

Enns, L.C., Kanaoka, M.M., Torii, K.U., Comai, L., Okada, K. and Cleland, R.E. (2005) Two callose synthases, GSL1 and GSL5, play an essential and redundant role in plant and pollen development and in fertility. Plant Mol. Biol. 58, 333-349.

Ferguson, C., Teeri, T.T., Siika-aho, M., Read, S.M. and Bacic, A. (1998) Location of cellulose and callose in pollen tubes and grains of Nicotiana tabacum. Planta, 206, 452-460.

Gardiner, J.C., Taylor, N.G. and Turner, S.R. (2003) Control of cellulose synthase complex localization in developing xylem. Plant Cell, 15, 1740-1748.

Hong, Z., Delauney, A.J. and Verma, D.P. (2001) A cell plate-specific callose synthase and its interaction with phragmoplastin. Plant Cell, 13, 755-768.

Honys, D. and Twell, D. (2004) Transcriptome analysis of haploid male gametophyte development in Arabidopsis. Genome Biol. 5, R85.

Howden, R., Park, S.K., Moore, J.M., Orme, J., Grossniklaus, U. and Twell, D. (1998) Selection of T-DNA-tagged male and female gametophytic mutants by segregation distortion in Arabidopsis. Genetics, 149, 621-631.

Jacobs, A.K., Lipka, V., Burton, R.A., Panstruga, R., Strizhov, N., Schulze-Lefert, P. and Fincher, G.B. (2003) An Arabidopsis callose synthase, GSL5, is required for wound and papillary callose formation. Plant Cell, 15, 2503-2513.

Kjell, J., Rasmusson, A.G., Larsson, H. and Widell, S. (2004) Protein complexes of the plant plasma membrane resolved by Blue Native PAGE. Physiol. Plant. 121, 546-555.

Levin, D.E. (2005) Cell wall integrity signaling in Saccharomyces cerevisiae. Microbiol. Mol. Biol. Rev. 69, 262-291.
Li, H.J., Bacic, A. and Read, S.M. (1997) Activation of pollen tube callose synthase by detergents - evidence for different mechanisms of action. Plant Physiol. 114, 1255-1265.

Li, J., Burton, R.A., Harvey, A.J., Hrmova, M., Wardak, A.Z., Stone, B.A. and Fincher, G.B. (2003) Biochemical evidence linking a putative callose synthase gene with $(1 \rightarrow 3)$ - $\beta$-D-glucan biosynthesis in barley. Plant Mol. Biol. 53, 213-225.

Mazur, P. and Baginsky, W. (1996) In vitro activity of 1,3- $\beta$-D-glucan synthase requires the GTP-binding protein Rho1. J. Biol. Chem. 271, 14604-14609.

Mazur, P., Morin, N., Baginsky, W., el-Sherbeini, M., Clemas, J.A., Nielsen, J.B. and Foor, F. (1995) Differential expression and function of two homologous subunits of yeast 1,3- $\beta$-D-glucan synthase. Mol. Cell. Biol. 15, 5671-5681.

McCormick, S. (1993) Male gametophyte development. Plant Cell, $\mathbf{5}$ 1265-1275.

Nishikawa, S., Zinkl, G.M., Swanson, R.J., Maruyama, D. and Preuss, D. (2005) Callose ( $\beta-1,3$ glucan) is essential for Arabidopsis pollen wall patterning, but not tube growth. BMC Plant Biol. 5,22

Nishimura, M.T., Stein, M., Hou, B.H., Vogel, J.P., Edwards, H. and Somerville, S.C. (2003) Loss of a callose synthase results in salicylic acid-dependent disease resistance. Science, 301, 969-972.

Oh, S.A., Johnson, J.A., Smertenko, A., Rahman, D., Park, S.K., Hussey, P.J. and Twell, D. (2006) A divergent cellular role for the FUSED kinase family in the plant-specific cytokinetic phragmoplast. Curr. Biol. 15, 2107-2111.

Ostergaard, L., Petersen, M., Mattsson, O. and Mundy, J. (2002) An Arabidopsis callose synthase. Plant Mol. Biol. 49, 559-566.

Park, S.K. and Twell, D. (2001) Novel patterns of ectopic cell plate growth and lipid body distribution in the Arabidopsis gemini pollen 1 mutant. Plant Physiol. 126, 899-909.

Park, S.K., Howden, R. and Twell, D. (1998) The Arabidopsis thaliana gametophytic mutation gemini pollen1 disrupts microspore polarity, division asymmetry and pollen cell fate. Development, 125, 3789-3799.

Pelosi, L., Imai, T., Chanzy, H., Heux, L., Buhler, E. and Bulone, V. (2003) Structural and morphological diversity of $(1 \rightarrow 3)-\beta$-Dglucans synthesized in vitro by enzymes from Saprolegnia monoica. Comparison with a corresponding in vitro product from blackberry (Rubus fruticosus). Biochemistry, 42, 6264-6274.

Rate, D.N., Cuenca, J.V., Bowman, G.R., Guttman, D.S. and Greenberg, J.T. (1999) The gain-of-function Arabidopsis acd6 mutant reveals novel regulation and function of the salicylic acid signaling pathway in controlling cell death, defenses, and cell growth. Plant Cell, 11, 1695-1708.

Rosso, M.G., Li, Y., Strizhov, N., Reiss, B., Dekker, K. and Weisshaar, B. (2003) An Arabidopsis thaliana T-DNA mutagenized population (GABI-Kat) for flanking sequence tag-based reverse genetics. Plant Mol. Biol. 53, 247-259.

Samuels, A.L., Giddings, T.H., Jr and Staehelin, L.A. (1995) Cytokinesis in tobacco BY-2 and root tip cells: a new model of cell plate formation in higher plants. J. Cell Biol. 130, 1345-1357.

Saxena, I.M. and Brown, R.M., Jr (2000) Cellulose synthases and related enzymes. Curr. Opin. Plant Biol. 3, 523-531.

Scherp, P., Grotha, R. and Kutschera, U. (2001) Occurrence and phylogenetic significance of cytokinesis-related callose in green algae, ferns and seed plants. Plant Cell Rep. 20, 143-149.

Schimoler-O'Rourke, R., Renault, S., Mo, W. and Selitrennikoff, C.P. (2003) Neurospora crassa FKS protein binds to the $(1,3) \beta$ glucan synthase substrate, UDP-glucose. Curr. Microbiol. 46, 408-412.

Stone, B.A. and Clarke, A.E. (1992) Chemistry and physiology of higher plant 1,3- $\beta$-glucans (Callose). In Chemistry and Biology of 
(1-3)- $\beta$-Glucans (Stone, B.A. and Clarke, A.E., eds). Melbourne: La Trobe University Press, pp. 365-429.

Taylor, N.G., Laurie, S. and Turner, S.R. (2000) Multiple cellulose synthase catalytic subunits are required for cellulose synthesis in Arabidopsis. Plant Cell, 12, 2529-2540.

Twell, D., Park, S.K., Hawkins, T.J., Schubert, D., Schmidt, R., Smertenko, A. and Hussey, P.J. (2002) MOR1/GEM1 has an essential role in the plant-specific cytokinetic phragmoplast. Nat. Cell Biol. 4, 711-714.

Twell, D., Oh, S.A. and Honys, D. (2006) Pollen development, a genetic and transcriptomic view. In The Pollen Tube: A Cellular and Molecular Perspective (Malho, R., ed.). Berlin: SpringerVerlag, pp. $15-45$.

Vanacker, H., Lu, H., Rate, D.N. and Greenberg, J.T. (2001) A role for salicylic acid and NPR1 in regulating cell growth in Arabidopsis. Plant J. 28, 209-216.

Yang, S. and Hua, J. (2004) A haplotype-specific resistance gene regulated by BONZAl1 mediates temperature-dependent growth control in Arabidopsis. Plant Cell, 16, 1060-1071.

Zimmermann, P., Hirsch-Hoffmann, M., Hennig, L. and Gruissem, W. (2004) GENEVESTIGATOR. Arabidopsis microarray database and analysis toolbox. Plant Physiol. 136, 2621-2632. 